\title{
Analysis of structure, surface morphology, optical and electrical properties of copper nanoparticles
}

\begin{abstract}
Copper $(\mathrm{Cu})$ nanoparticles were prepared by chemical reduction method. The synthesized $\mathrm{Cu}$ nanoparticles were characterized with different techniques such as powder X-ray diffraction (XRD), Scanning Electron Microscopy (SEM), Energy Dispersive X-ray (EDX) spectrum, Transmission electron microscopy (TEM), UV-Visible spectrum and the dielectric properties of the prepared nanoparticles. The X-ray diffraction (XRD) analysis was used to study the structure and crystallite size of $\mathrm{Cu}$ nanoparticles. The morphology and the size of the $\mathrm{Cu}$ nanoparticles were characterized using Scanning Electron Microscopy and Transmission Electron Microscopy (SEM and TEM). The elemental composition of $\mathrm{Cu}$ nanoparticles was analyzed by Energy Dispersive X-ray (EDX) spectrum. The particle size of the $\mathrm{Cu}$ nanoparticles was also analyzed, using the Dynamic Light Scattering (DLS) experiment. The optical properties were studied using the UV-Visible spectrum in the wavelength range of $550-900 \mathrm{~nm}$. The dielectric properties of $\mathrm{Cu}$ nanoparticles were studied at room temperature.
\end{abstract}

Keywords: Cu Nanoparticles, XRD, SEM, TEM, UV analysis, Dielectric studies
Volume 2 Issue 5 - 2015

\author{
Suresh Sagadevan, ${ }^{\prime}$ Koteeswari $P^{2}$ \\ 'Department of Physics, AMET University, India \\ 2Department of Physics, Justice Basheer Ahmed Sayeed College \\ for Women, India
}

Correspondence: Suresh Sagadevan, Department of Physics, AMET University, Chennai-603 II 2, India, Tel 99528966 I5

Email sureshsagadevan@gmail.com

Received: October 27, 2015 | Published: December 08, 2015
Abbreviations:Cu, Copper; DLS, Dynamic Light Scattering; XRD, X-Ray Diffraction; SEM, Scanning Electron Microscopy; TEM, Transmission Electron Microscopy; EDX, Energy Dispersive X-ray; MNPs, Metallic Nanoparticles; AR, Analytical Grade

\section{Introduction}

Nanoscience and nanotechnology involve the study and working with matter on an ultra-small scale. Nanomaterial is of enormous scientific attention as they are effectively a bridge between bulk materials and atomic or molecular structures. A bulk material must have constant physical properties apart from of its size, but at nano scale this is often not the case. The properties of the materials modify as their size approaches the nano scale and as the percentage of atoms at the surface of a material suits important. For bulk materials bigger than one micrometer the percentage of atoms at the surface is less, relative to the total number of atoms of the material. Therefore these interesting and sometimes unexpected properties are partly due to the surface that leads in nanoparticles, which is not the case in bulk materials. Interest in metal nanoparticles is driven which reveal novel chemical and physical properties due to their small physical dimensions. Owing to their small size and large surface area the metal nanoparticles have unique electronic, mechanical, magnetic and chemical properties that are different from those of bulk materials. For these reasons metallic nanoparticles have found applications in different fields such as electronic, thermal, catalysis, photonics, biosensors and optoelectronics. ${ }^{1-4}$

Metallic nanoparticles (MNPs) were the subject of deep research during the recent years because of their unique properties compared to bulk metals. ${ }^{5-7}$ Among the different metal particles, copper nanoparticles have received considerable attention because copper is a versatile material which finds various applications in different fields of research. Copper has a superb electrical conductivity. Owing to fairly low costs, copper plays a significant role in modern electronic circuits. ${ }^{8}$ Because of its admirable electrical conductivity, catalytic behaviour, good compatibility and surface enhanced Raman scattering activity, copper nanoparticles have drawn the attention of scientists who will be using them as components in the nano- devices of future. ${ }^{9}$ Copper nanoparticles were investigated to be used as nanoprobes in medicines and bio-analytical areas. Copper nanoparticles and nanowires were prepared by different methods. ${ }^{10-12}$ Copper nanoparticles are broadly used as alternative catalysts, IR such as selective hydrogenation and methanol synthesis reactions, which make them suitable for application in the field of catalysis and it is also extensively used in lubricants, polymers/plastic, metallic coating and ink. ${ }^{13,14}$ The prepared copper nanoparticles were characterized by powder X-ray diffraction analysis, Scanning Electron Microscopy (SEM), Transmission Electron Microscopy (TEM), Energy Dispersive X-ray (EDX) spectrum, DLS, UV-analysis and dielectric studies.

\section{Experimental procedure}

In a typical synthesis, Copper sulphate, sodium borohydride and tri-sodium citrate of analytical grade (AR) purity were used. The solutions of all the reacting materials were prepared in distilled water. Copper sulphate solution kept in a flask was reduced by drop wise addition of highly dilute solution of sodium borohydride in a nitrogen atmosphere. During the process of reaction, the solution was stirred forcefully. As the colour of the solution turned to light yellow $5 \mathrm{ml}$ of trisodium citrate was added drop by drop as stabilizer. After the solutions were cooled to room temperature, nanoparticles were obtained by centrifugation and washing. The as-prepared nanoparticles were dried in vacuum at $60^{\circ} \mathrm{C}$ for 12 hours for characterization.

\section{Results and Discussion}

\section{$X$-ray diffraction analysis}

$\mathrm{X}$-ray powder diffraction (XRD) is a powerful technique used to uniquely identify the crystalline phases present in materials and to measure the structural properties (strain state, grain size, epitaxy, phase composition, preferred orientation, and defect structure) of these phases. The XRD pattern of the $\mathrm{Cu}$ nanoparticles was recorded by using a powder X-ray diffractometer (Schimadzu model: XRD 6000 using $\mathrm{CuK} \alpha$ ) with a diffraction angle between $20^{\circ}$ and $80^{\circ}$. Figure 1 shows X-ray diffraction patterns of Copper nanoparticles. The broadenings of the peaks due to crystallites with sizes in the nanometer scale are also observed. The peaks are very sharp due to the nanocrystalline nature of copper. Figure 1 shows three peaks (111), 
(200) and (220) planes of copper, respectively. No impurity peak is observed in the X-ray diffraction pattern. The crystallite size was determined from the broadenings of the corresponding X-ray spectral peaks by using Scherrer's formula. The average nano-crystalline size (D) was calculated using the Scherrer formula,

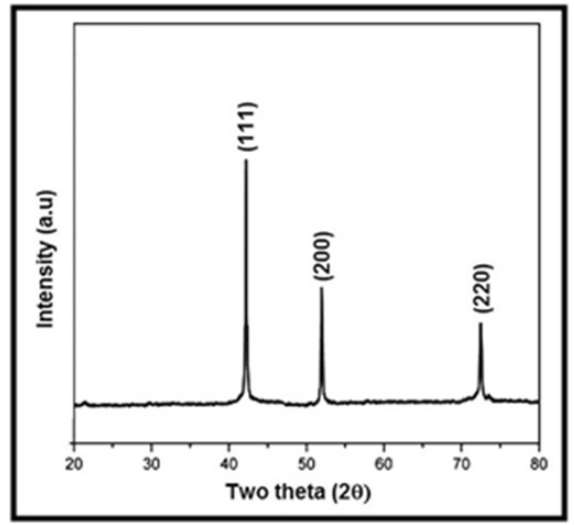

Figure I XRD spectrum of Cu nanoparticles.

$$
D=\frac{0.9 \lambda}{\beta \cos \theta}^{(1)}
$$

Where $\lambda$ is the $\mathrm{X}$-ray wavelength, $\theta$ is the Bragg diffraction angle, and $\beta$ is the FWHM of the XRD peak appearing at the diffraction angle $\theta$. The average crystalline size was calculated from X-ray line broadening peak and Scherrer equation and it was found to be about $22 \mathrm{~nm}$.

\section{Scanning electron microscopy (SEM) and energy- dispersive $\mathbf{X}$-ray (EDX) analysis}

Scanning electron microscope (SEM) is one of the most widely used techniques used in characterization of nanomaterials and nanostructures. The signals that derive from electron-sample interactions reveal information about the sample including surface morphology (texture), chemical composition of the sample. The morphology of the $\mathrm{Cu}$ nanoparticles was carried out on JEOL, JSM67001 and the image is shown in Figure 2. The average particle size of the $\mathrm{Cu}$ nanoparticles was obtained around $50 \mathrm{~nm}$ and they were of spherical shape. The composition of copper nanoparticles was analyzed by using Energy-Dispersive X-ray (EDX) analysis and the image is shown in Figure 3. The spectrum shows sharp peaks that confirm the presence of $\mathrm{Cu}$ nanoparticles.

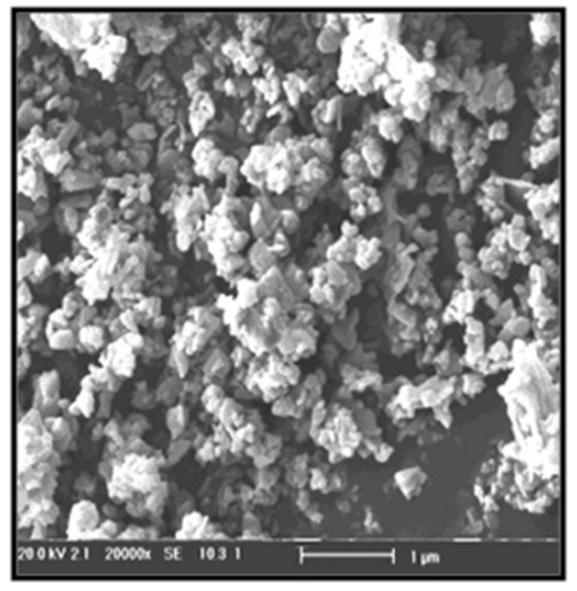

Figure 2 SEM image of Cu nanoparticles.

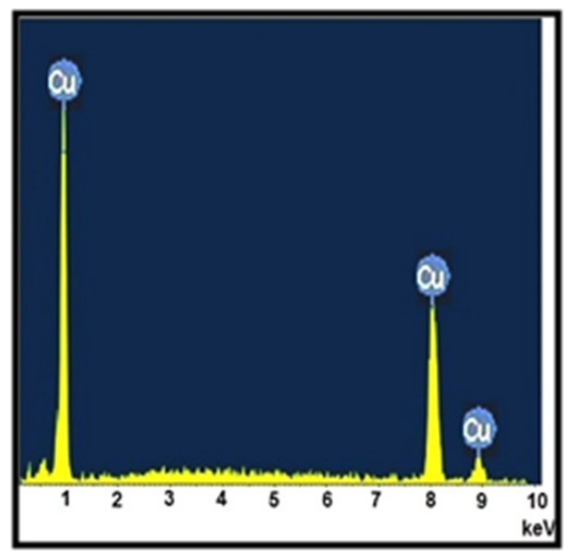

Figure 3 EDX image of Cu nanoparticles.

\section{Transmission electron microscopy (TEM)}

Transmission electron microscopy (TEM) is widely used to observe the size of small nanoparticles through imaging, revealing phase/crystallographic orientation information through a diffraction pattern and discovering chemical composition by means of the energy spectrum. Transmission Electron Microscope (TEM) image was obtained using an H-800 TEM (Hitachi, Japan) with an accelerating voltage of $100 \mathrm{kV}$ TEM is commonly used for imaging and analytical characterization of the nanoparticles to assess the shape, size, and morphology. TEM image of the as-prepared spherical shaped $\mathrm{Cu}$ nanoparticles is shown in Figure 4. In addition to the individual particles, some aggregates are also present. The particle sizes are estimated in the range of 30 to $50 \mathrm{~nm}$.

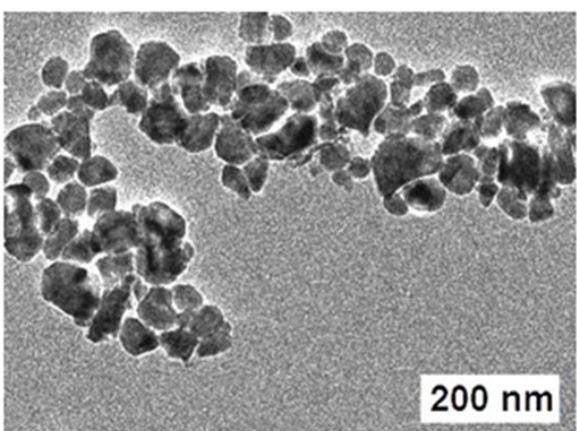

Figure 4 TEM image of $\mathrm{Cu}$ nanoparticles.

\section{Dynamic light scattering (DLS) studies}

Dynamic Light Scattering (DLS) is a very important tool for characterizing the size of nanoparticles in a solution. When the DLS sizing data are compared with the transmission electron microscope images, the aggregation state of the particles can be analyzed. The dynamic light scattering experiment showed that the particle size of copper nanoparticles was in the range of 30 to $50 \mathrm{~nm}$, and this was well supported by the TEM analysis (Figure 5).

\section{Optical studies}

Absorption of electromagnetic radiation is the way by which the energy of a photon is taken up by matter, typically the electrons of an atom. UV-visible spectroscopy is used when involving the absorption of these high energy lights by atoms or molecules, which causes electronic excitation. The optical absorption spectrum of copper nanoparticles was recorded in the wavelength region $550-900 \mathrm{~nm}$ using the VARIAN CARY MODEL 5000 spectrophotometer and it is shown in Figure 6a. The synthesized copper nanoparticles show 
an absorption peak at around $570 \mathrm{~nm}$. This peak can be ascribed to the absorption of nanoparticles of copper. The dependence of optical absorption coefficient on photon energy helps to analyze the band structure and the type of transition of electrons. The optical absorption coefficient $(\alpha)$ was calculated from transmittance using the following relation

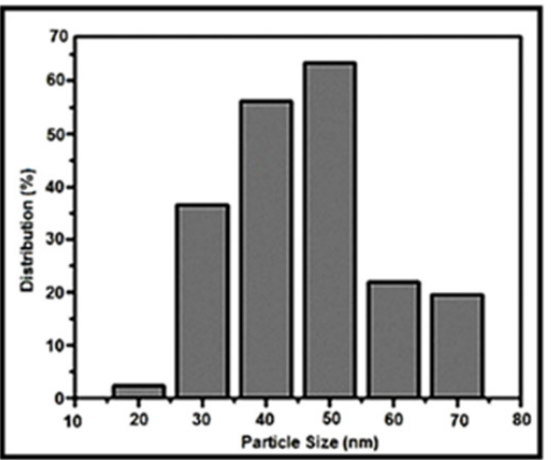

Figure 5 Particle size of Cu nanoparticles.

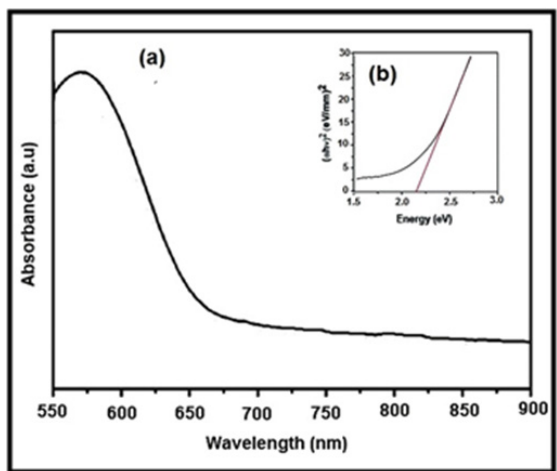

Figure 6a\&b UV-Visible absorption spectrum of Cu nanoparticles (b) Plot of $(\alpha h v)^{2}$ vs photon energy.

$$
\alpha=\frac{1}{d} \log \left[\frac{1}{T}\right]
$$

Where $\mathrm{T}$ is the transmittance and $\mathrm{d}$ is the thickness of the sample. The study has an absorption coefficient $(\alpha)$ obeying the following relation for high photon energies $(\mathrm{h} v)$

$$
\alpha=\frac{A\left(h v-E_{g}\right)^{1 / 2}}{h v}
$$

where $\alpha, \mathrm{E}_{\mathrm{g}}$ and $\mathrm{A}$ are the absorption coefficient, band gap and constant respectively. Determination of optical band gap is based on the photon induced electronic transition between the conduction band and the valance band. By extrapolating the linear region in the plots of versus $(\alpha h v)^{2}$ versus $h v$ is shown in Figure 6b, the band gap value is estimated at $2.3 \mathrm{eV}$.

\section{Dielectric studies}

The dielectric analysis is an important tool used to know the details about the electrical properties of material at different frequencies. The dielectric properties of the copper nanoparticles were analyzed using a HIOKI 3532-50 LCR HITESTER over the frequency range $50 \mathrm{~Hz}-5 \mathrm{MHz}$. The dielectric constant was analyzed as a function of the frequency at room temperature as shown in Figure 7, while the corresponding dielectric loss is shown in Figure 8. It is observed from the profile that both the dielectric constant and the dielectric loss decrease with increase in frequency at room temperature. The large dielectric constant at low frequency shows the occurrence of space charge polarization arising at the grain boundary interface..$^{15,16}$ The involvement of the decrease in the dielectric constant owing to electronic polarization is relatively less. The contribution to polarizability of the space charge depends on the purity of the nanoparticles. ${ }^{17}$ The dielectric loss studied as a function of frequency at room temperature is shown in Fig.8. The dielectric loss decreases with an increase in the frequency. The curve suggests that the dielectric loss is strongly dependent on the frequency of the applied field, comparable to that of the dielectric constant.

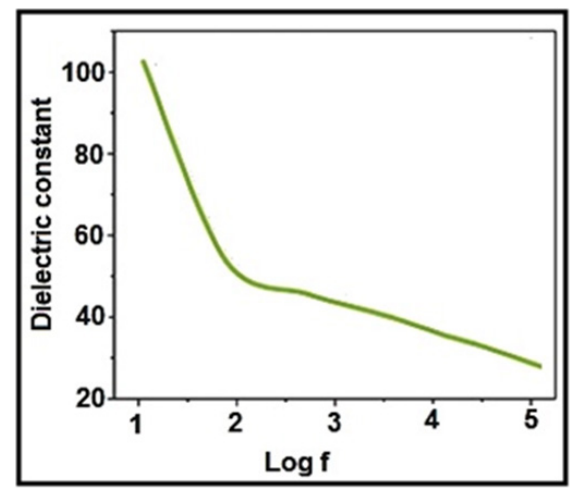

Figure 7 Dielectric constant of $\mathrm{Cu}$ nanoparticles.

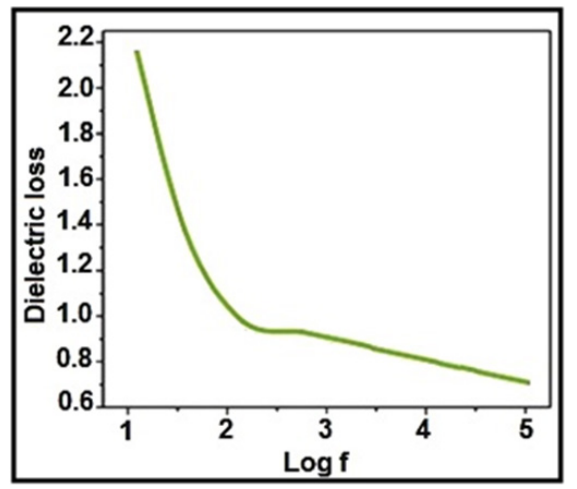

Figure 8 Dielectric loss of Cu nanoparticles.

\section{Conclusion}

Copper nanoparticles were prepared by chemical reduction method. Their structural properties were investigated by XRD analysis. The average crystallite size of copper nanoparticles was calculated from the X-ray diffraction (XRD) pattern and found to be $22 \mathrm{~nm}$. The scanning electron microscopy (SEM) analysis showed that the nanoparticles agglomerated forming spherical-shaped particles. The TEM results revealed that the particle size measured in the range of 30 to $50 \mathrm{~nm}$. The particle size of the copper nanoparticles lying in the range 30 to $50 \mathrm{~nm}$ was determined using the dynamic light scattering (DLS) experiment which agreed well with the results of the TEM analysis. The optical properties were studied by the UV-Visible spectrum and the band gap value was found to be $2.3 \mathrm{eV}$. The dielectric constant, dielectric loss of copper nanoparticles also investigated as a function of frequency at room temperature. It indicates that the dielectric constant and dielectric loss of the materials decreases with increasing frequency.

\section{Acknowledgments}

None. 


\section{Conflicts of interest}

None.

\section{References}

1. Daniel MC, Astruc D Gold nanoparticles: assembly, supramolecular chemistry, quantum-size-related properties, and applications toward biology, catalysis, and nanotechnology. Chem Rev. 2004;104(1):293-346.

2. Tian Y, Tatsuma T Plasmon-induced photoelectrochemistry at metal nanoparticles supported on nanoporous TiO2. Chem Commun. 2004;1810-1811.

3. Kalidindi SB, Sanyal U, Jagirdar BR Nanostructured $\mathrm{Cu}$ and $\mathrm{Cu} @ \mathrm{Cu} 2 \mathrm{O}$ core shell catalysts for hydrogen generation from ammonia-boranew. Phys Chem Chem Phys. 2008;10:5870-5874.

4. Grzelczak M, Juste JP, Mulvaney P, Marzan LML Shape control in gold nanoparticle synthesis. Chem Soc Rev. 2008;37:1783-1791.

5. Dong $\mathrm{C}$, Zhou $\mathrm{K}$, Zhang $\mathrm{X}$ et al. Semen cassiae extract mediated novel route for the preparation of silver nanoparticles. Mater Lett. 2014;120:118-121.

6. Zhou K, Dong C, Zhang X et al. Preparation and characterization of nanosilver-doped porous hydroxyapatite scaffolds. Ceram Int. 2015;41(1):1671-1676.

7. Adil SF, Assal ME, Khan M et al. Biogenic synthesis of metallic nanoparticles and prospects toward green chemistry. Dalton Trans. 2015;44:9709-9717.

8. Sulekh Chandra, Avdhesh Kumar, Praveen Kumar T Synthesis and characterization of copper nanoparticles by reducing agent. Journal of Saudi Chemical Society. 2014;18(2):149-153.
9. Pergolese B, Miranda Muniz M, Bigotto A Synthesis and characterization of copper nanoparticles by reducing. J Phys Chem B. 2006;110:9241-9247.

10. Hori $\mathrm{H}$, Yamamoto $\mathrm{Y}$, Iwamoto $\mathrm{T}$ et al. Diameter dependence of ferromagnetic spin moment in Au nanocrystals. Phys Rev B. 2004;69:174411-174416.

11. Crespo P, Litran R, Rojas TC et al. Permanent Magnetism, Magnetic Anisotropy, and Hysteresis of Thiol-Capped Gold Nanoparticles. Phys Rev Lett. 2004;93:087204-087207.

12. Craik DJ Magnetism Principles and Applications. John Wiley \& Sons, New York, USA, p: 1995;1-72.

13. Setlur AA, Lauerhaas JM, Dai JY et al. A method for synthesizing large quantities of carbon nanotubes and encapsulated copper nanowires. Appl Phys Lett. 1996;69(3):345-347.

14. Mane RS, Lokhande CD Chemical deposition method for metal chalcogenide thin films. Mater Chem Phys. 2000;65(1):1-31.

15. Suresh $\mathrm{S}$ Wet chemical synthesis of Tin Sulfide nanoparticles and its characterization. International Journal of Physical Sciences. 2014;9(17):380-385.

16. Suresh S, Arunseshan C Dielectric Properties of Cadmium Selenide (CdSe) Nanoparticles synthesized by solvothermal method. Appl Nanosci. 2014;4(2):179-184.

17. Sagadevan Suresh Studies on the dielectric properties of CdS nanoparticles. Appl Nanosci. 2014;4(3):325-329. 